\title{
Impact of Dexmedetomidine On Secondary Hyperparathyroidism Recurrence In Uremic Patients Who Received Parathyroidectomy With Auto-Transplantation: A Retrospective Propensity-Matched Study
}

\author{
Yun Wu \\ The Second Affiliated Hospital of Anhui Medical University \\ Wei Han \\ The Second Affiliated Hospital of Anhui Medical University

\section{Peikun Li} \\ The Second Affiliated Hospital of Anhui Medical University \\ Xianwen $\mathrm{Hu}$ \\ The Second Affiliated Hospital of Anhui Medical University \\ Ye Zhang ( $\sim$ wuyunanyi@163.com ) \\ The Second Affiliated Hospital of Anhui Medical University
}

\section{Research Article}

Keywords: Dexmedetomidine, Secondary hyperparathyroidism, Recurrence, End-stage renal disease, Parathyroidectomy

Posted Date: December 6th, 2021

DOI: https://doi.org/10.21203/rs.3.rs-1115740/v1

License: (ㄷ) (i) This work is licensed under a Creative Commons Attribution 4.0 International License. Read Full License 


\section{Abstract}

Background: Recurrence of secondary hyperparathyroidism (SHPT) remains a big challenge in uremic patients who underwent total parathyroidectomy with auto-transplantation (tPTX-AT). However, the relationship between perioperative intervention and recurrence of SHPT remains unclear. Dexmedetomidine has been used safely and effectively in uremic patients' anesthesia. The aim of the study was to explore the effect of dexmedetomidine on the recurrence of SHPT and speculate the possible mechanism of action.

Methods: Records of patients who underwent tPTX-AT between 2017 and 2018 were retrospectively analyzed. The study consisted of patients who received dexmedetomidine intra-operatively and the controls were patients who did not receive dexmedetomidine. The primary endpoint was the difference in the recurrence of SHPT one year after surgery between the two groups. The secondary endpoint was health-related quality of life scores. Analysis included propensity score matching and multivariable logistic regression.

Results: Of 354 patients, 133 patients received dexmedetomidine intraoperatively, and the total recurrence rate of SHPT was $10.2 \%$. After propensity score matching, patients who received dexmedetomidine had a 3.80 -fold decreased risk of SHPT recurrence (odds ratio, $0.263 ; 95 \%$ confidence interval, 0.081 to $0.854 ; \mathrm{P}=0.026$ ) and exhibited a better quality of life in terms of physical functioning and general health, and less emotional role limitations compared with those in control group.

Conclusion: In uremic patients who received tPTX-AT, there was an association between dexmedetomidine use and decreased risk of SHPT recurrence. Further studies are needed to accurately assess the effects and mechanism of action of dexmedetomidine on the prognosis of this population.

\section{Introduction}

Secondary hyperparathyroidism (SHPT) is a common complication in uremic patients. It is characterized by markedly elevated intact parathyroid hormone (iPTH) concentrations due to persistent stimulation of the parathyroid tissue and resultant parathyroid hyperplasia in response to hypocalcemia [1]. Chronically elevated iPTH causes bone pain, fractures, cardiovascular disease, hematopoiesis, immune dysfunction, all of which may influence the length and quality of life (QOL) of uremic patients [2].

Surgical total parathyroidectomy with auto-transplantation (tPTX-AT) significantly improves calcium and phosphorus metabolism, resulting in reduced mortality and morbidity rates. However, due to the pathological changes in the remaining tissue, including inflammation and perioperative stress, the incidence of SHPT recurrence may reach as high as $20 \%[3,4]$. Many cohort studies have demonstrated that perioperative anesthetic and analgesic intervention play a critical role on the long-term prognosis of multiple disease $[5,6]$. Therefore, the anesthetic impact on the recurrenc of SHPT as well as quality of life in uremic patients after surgery need to be identified.

Dexmedetomidine (DEX), a highly selective alpha-2 adrenoceptor agonist, is increasingly used with the beneficial effects of analgesia, sympathetic tone inhibition, anti-inflammation and surgical stress [7-9]. DEX also exhibited potential renal protection in patients with renal disease [10]. However, there are no current studies focussing on the impact of DEX on the outcome in uremic patients after surgery. Therefore, we performed a single-centre retrospective study to evaluate the relationship between DEX use and SHPT recurrence in uremic patients undergoing IPTX-AT. Specifically, the primary aim was to test the hypothesis that infusion of DEX is associated with a lower incidence of SHPT recurrence. Second, we aimed to test the hypothesis that DEX infusion elevated both the postoperative short-term outcome and long-term QOL in uremic patients. 


\section{Study Design And Methods}

The study protocol (PJ-YX2019-049F2) was reviewed and approved by Ethics Committee for Clinical Trials of the Second Affiliated Hospital of Anhui Medical University, Anhui, China (Chairperson Prof. Chao Lu) on 8th April 2020, which waived the requirement for written informed consent because all data were de-identified and handled anonymously. The study was registered at the Chinese Clinical Trial Registry (ChiCTR-2000033811). All the work was done in accordance with the Ethical Principles for Medical Research Involving Human Subjects outlined in the Declaration of Helsinki.

Data were collected from the electronic medical records of all uremic patients who underwent tPTX-AT for SHPT by the same surgical team at the Second Affiliated Hospital of Anhui Medical University from January 2017 to August 2018. Patients were excluded if they met any of the following criteria: an American Society of Anesthesiologists (ASA) physical status of $\mathrm{V}$, primary or tertiary hyperparathyroidism, a history of a previous thyroid operation, operation performed by any other surgeons, and incomplete records for anesthesia or surgery.

All patients received propofol-sufentanil-based general anesthesia and were transferred to the post-anesthesia care unit (PACU) after tracheal extubation in the operating room. DEX use was defined as a bolus infusion of 0.5 to $1 \mu \mathrm{g} \mathrm{kg}-1$ for $10 \mathrm{~min}$ before induction followed by infusion of 0.3 to $0.5 \mu \mathrm{g} \mathrm{kg}^{-1} \mathrm{~h}^{-1}$ intra-operatively and ceased about 30 min before the end of the surgery. Patients were divided into two groups: those who received DEX (DEX group) and those who did not (control, CON group).

The patients' characteristics included age, sex, body mass index, comorbidities, physical ASA status, serum concentrations of calcium, phosphorus, iPTH, and alkaline phosphatase at baseline, dialysis modality, and the length of dialysis. Surgical information included the year of surgery, duration of anesthesia, consumption of sufentanil, extubation time, length of PACU stay, comorbidities in the PACU, length of hospitalisation after surgery, and the concentrations of postoperative calcium, phosphorus, and IPTH at discharge. Patients were followed up for one year after the surgery. Follow-up assessments included the concentrations of iPTH concentrations,

C-reactive protein (CRP) and a self-administered questionnaire on the health-related QOL, which was measured by a validated Chinese version of the 36-item Short Form Health Survey (SF-36) [11, 12] as a generic core. The SF-36 was developed to assess 8 different aspects of the physical and mental health status: physical functioning (10 items), physical role limitation (4 items), emotional role limitation (3 items), social functioning (2 items), mental health (5 items), bodily pain ( 2 items), vitality ( 4 items), and general health perception ( 5 items). Each component was analyzed individually as the average of a predefined sum of questions, ranging from 0 to 3 or from 0 to 5 , depending on the number of possible answers. Each response was linearly transformed to 0 to 100 , with higher scores indicating a better QOL.

\section{Statistical Analysis}

In order to correct for selection bias and confounding factors, we used the propensity score matching method without replacement, which could balance the covariates between the two groups. The following covariates were matched at a $1: 2$ ratio with a 0.03 calliper by the nearest neighbour method: patient characteristics at baseline, preoperative comorbidities, ASA physical status, modality of dialysis, length of dialysis, year of surgery, duration of anesthesia. To determine the balance between the two groups before and after propensity score matching, absolute standardized mean difference (ASD) was used; an ASD < 0.1 for the covariates indicated that the two groups were sufficiently balanced. 
Continuous variables were reported as mean \pm SD or median [IQR], and categorical variables were reported as number (\%). Data with normal distribution were compared using independent-samples $t$ test. For data that did not have normal distribution, the rank sum test was used. For categorical data, Pearson's $\chi^{2}$ test was used. Potential confounders associated with recurrence after TPTX-AT, which were chosen on the basis of their clinical significance as reported in the literature, were analysed using univariate and multivariable logistic regression. First, we performed a univariate analysis to identify potential risk factors for postoperative recurrence. Variables with $P$-values $<0.5$ were subjected to multivariable analysis, after which, the odds ratios (OR) and associated $95 \%$ confidence intervals (CI) were calculated. The multivariable logistic regression processes were additively adjusted for several potential confounding. Multivariable associations between DEX administration and postoperative recurrence were also assessed by logistic regression analyses.

The study was powered to evaluate one primary outcome, the recurrence of SHPT one year after in uremic patients. According to the previous study [3] and the historical medical data in our hospital, we hypothesized that uremic patients would have a $10 \%$ rate of recurrent SHPT. To detect a $4 \%(S D=3 \%)$ difference in the incidence of SHPT recurrence between patients received or did not receive DEX with a 0.05 chance of type $\nabla$ error and $80 \%$ power, 304 patients were required.

The package of the R program (version 2.15.X) was used as a propensity score matching tool; the analysis was performed with SPSS software version 22.0 (SPSS Inc., Chicago, IL). A $P$-value $<0.05$ was considered statistically significant.

\section{Results}

\section{Patients}

Among the 399 uremic patients who underwent tPTX-AT between January 2017 and August 2018, we excluded 18 with an ASA status of V, two with tertiary hyperparathyroidism, nine with a history of previous thyroid operation, thirteen with tPTX-AT by other surgeons, and 3 with incomplete or missing records for surgery. Finally, 354 patients were included in the analyses (Fig. 1). One year after the surgery, recurrence occurred in 36 patients (10.2\%) (Table 1). 
Table 1

Characteristics of patients who received dexmedetomidine (DEX group) and those who did not receive dexmedetomidine (CON group).

\begin{tabular}{|c|c|c|c|c|c|c|}
\hline \multirow[t]{3}{*}{ Parameters } & \multicolumn{3}{|c|}{ Before propensity score matching $(n=354)$} & \multicolumn{3}{|c|}{$\begin{array}{l}\text { After } 1: 2 \text { propensity score matching } \\
(n=268)\end{array}$} \\
\hline & CON group & DEX group & ASD & CON group & DEX group & ASD \\
\hline & $n=221$ & $n=133$ & & $n=157$ & $n=111$ & \\
\hline Recurrence & $30(13.6)$ & $6(4.5)$ & 0.312 & 19(12.1) & $5(4.5)$ & 0.273 \\
\hline Age (years) & $46.9 \pm 9.6$ & $48.1 \pm 9.5$ & 0.133 & $47.4 \pm 9.6$ & $47.3 \pm 9.2$ & 0.006 \\
\hline$<40$ & $45(20.4)$ & $25(18.8)$ & & $29(18.5)$ & $23(20.7)$ & \\
\hline 40 to 50 & $87(39.4)$ & $51(38.3)$ & & $60(38.2)$ & $44(39.6)$ & \\
\hline 50 to 60 & $71(32.1)$ & $40(30.1)$ & & $55(35.0)$ & $32(28.8)$ & \\
\hline 60 to 70 & $15(6.8)$ & 15(11.3) & & $11(7.0)$ & $11(9.9)$ & \\
\hline$\geq 70$ & $3(1.4)$ & $2(1.5)$ & & $2(1.3)$ & $1(0.9)$ & \\
\hline Female & $92(41.6)$ & $52(39.1)$ & 0.052 & $66(42.0)$ & $44(39.6)$ & 0.028 \\
\hline $\mathrm{BMI}\left(\mathrm{kg} \mathrm{m}^{-2}\right)$ & $\begin{array}{l}21.83[19.72 \text { to } \\
24.43]\end{array}$ & $\begin{array}{l}21.78[19.82 \text { to } \\
24.22]\end{array}$ & 0.063 & $\begin{array}{l}22.06[19.88 \\
\text { to } 24.74]\end{array}$ & $\begin{array}{l}21.68[19.59 \\
\text { to } 24.09]\end{array}$ & 0.059 \\
\hline$<18.5$ & $33(14.9)$ & $15(11.3)$ & & $21(13.4)$ & 13(11.7) & \\
\hline 18.5 to 25 & $143(64.7)$ & $97(72.9)$ & & $102(65.0)$ & $82(73.9)$ & \\
\hline 25 to 30 & $41(18.6)$ & $18(13.5)$ & & $32(20.4)$ & $14(12.6)$ & \\
\hline$\geq 30$ & $4(1.8)$ & $3(2.3)$ & & $2(1.3)$ & $2(1.8)$ & \\
\hline \multicolumn{7}{|l|}{ Commoribidities } \\
\hline Hypertension & 186(84.2) & $114(85.7)$ & 0.044 & 135(86.0) & $94(84.7)$ & 0.026 \\
\hline Diabetes & $6(2.7)$ & $3(2.3)$ & 0.031 & $5(3.2)$ & $3(2.7)$ & 0.030 \\
\hline $\begin{array}{l}\text { Cardiopulmonary } \\
\text { disease }\end{array}$ & $13(5.9)$ & $5(3.8)$ & 0.111 & $6(3.8)$ & $5(4.5)$ & 0.047 \\
\hline \multicolumn{7}{|l|}{$\begin{array}{l}\text { ASA physical } \\
\text { status }\end{array}$} \\
\hline ( & 203(91.9) & 127(95.5) & 0.174 & 152(96.8) & 105(94.6) & 0.086 \\
\hline$\square$ & $18(8.1)$ & $6(4.5)$ & & $5(3.2)$ & $6(5.4)$ & \\
\hline \multicolumn{7}{|l|}{ Type of dialysis } \\
\hline Hemodialysis & $217(98.2)$ & 132(99.2) & 0.122 & 156(99.4) & 111(100.0) & 0.052 \\
\hline Peritoneal dialysis & $4(1.8)$ & $1(0.8)$ & & $1(0.6)$ & $0(0)$ & \\
\hline
\end{tabular}

Data are presented as the mean \pm SD or median [IQR] for continuous variables, and number (\%) for categorical variables.

Abbreviations: ASD, absolute standardized mean difference; BMI, body mass index; ASA, American Society of Anesthesiologist; Ca, calcium; P, phosphorus; iPTH, intact parathyroid hormone; ALP, alkaline phosphatase. 


\begin{tabular}{|c|c|c|c|c|c|c|}
\hline \multirow{2}{*}{$\begin{array}{l}\text { Parameters } \\
\begin{array}{l}\text { Dialysis duration } \\
\text { (years) }\end{array}\end{array}$} & \multicolumn{3}{|c|}{ Before propensity score matching ( $n=354)$} & \multicolumn{3}{|c|}{$\begin{array}{l}\text { After } 1: 2 \text { propensity score matching } \\
(n=268)\end{array}$} \\
\hline & $7.7 \pm 3.1$ & $8.2 \pm 3.0$ & 0.170 & $7.9 \pm 3.0$ & $8.0 \pm 2.7$ & 0.007 \\
\hline $\begin{array}{l}\text { Duration of } \\
\text { anesthesia (min) }\end{array}$ & $\begin{array}{l}116.0[92.5 \text { to } \\
152.0]\end{array}$ & $\begin{array}{l}120.0[89.0 \text { to } \\
152.5]\end{array}$ & 0.004 & $\begin{array}{l}110.0[90.0 \text { to } \\
147.5]\end{array}$ & $\begin{array}{l}120.0[89.0 \text { to } \\
155.0]\end{array}$ & 0.005 \\
\hline \multicolumn{7}{|l|}{ Year at surgery } \\
\hline 2017 & $113(51.1)$ & $74(55.6)$ & 0.090 & $80(51.0)$ & $60(54.1)$ & 0.027 \\
\hline 2018 & $108(48.9)$ & $59(44.4)$ & & $77(49.0)$ & $51(45.9)$ & \\
\hline \multicolumn{7}{|l|}{$\begin{array}{l}\text { Preoperative } \\
\text { measures }\end{array}$} \\
\hline $\begin{array}{l}\text { Serum Ca (mg } \\
\left.\mathrm{dl}^{-1}\right)\end{array}$ & $2.36 \pm 0.26$ & $2.35 \pm 0.25$ & 0.020 & $2.37 \pm 0.28$ & $2.37 \pm 0.22$ & 0.014 \\
\hline Serum $P\left(\mathrm{mg} \mathrm{dl}^{-1}\right)$ & $2.10 \pm 0.54$ & $2.09 \pm 0.52$ & 0.011 & $2.10 \pm 0.54$ & $2.10 \pm 0.53$ & 0.018 \\
\hline $\begin{array}{l}\text { Serum iPTH (pg } \\
\left.\mathrm{ml}^{-1}\right)\end{array}$ & $\begin{array}{l}1768.0[1219.0 \\
\text { to } 2500.0]\end{array}$ & $\begin{array}{l}1791.0[1294.0 \\
\text { to } 2500.0]\end{array}$ & 0.008 & $\begin{array}{l}1790.0 \\
{[1218.0 \text { to }} \\
2500.0]\end{array}$ & $\begin{array}{l}1820.0 \\
{[1299.0 \text { to }} \\
2500.0]\end{array}$ & 0.019 \\
\hline Serum ALP $\left(U L^{-1}\right)$ & $\begin{array}{l}462.0[244.0 \text { to } \\
761.5]\end{array}$ & $\begin{array}{l}414.0[212.0 \text { to } \\
677.0]\end{array}$ & 0.233 & $\begin{array}{l}463.0[251.0 \\
\text { to } 761.0]\end{array}$ & $\begin{array}{l}414.0[229.0 \\
\text { to } 756.0]\end{array}$ & 0.042 \\
\hline \multicolumn{7}{|c|}{$\begin{array}{l}\text { Data are presented as the mean } \pm \text { SD or median [IQR] for continuous variables, and number }(\%) \text { for categorical } \\
\text { variables. }\end{array}$} \\
\hline
\end{tabular}

\section{Comparison of characteristics between patients who did and did not receive DEX}

Table 1 shows the pre-propensity score matching (DEX group: $n=133$; CON group: $n=221$ ) and post-propensity score matching (DEX group: $n=111$; CON group: $n=157$ ) covariate comparisons. After propensity score matching, all covariates were well-balanced with an ASD < 0.1 . The postoperative recurrence rate was $4.5 \%$ in the DEX group, which was significantly lower than that in the $\operatorname{CON}$ group $(13.6 \%, P=0.006)$, and the recurrence rate remained significantly lower in the DEX group after propensity score matching $(4.5 \%$ vs $12.1 \%, P=0.032)$.

\section{Association between DEX and postoperative recurrence of SHPT}

The factors associated with recurrence one year after IPTX-AT are presented in Table 2. After the univariate analysis, the covariates of patient characteristics at baseline and other relative parameters were adjusted in the multivariable analysis with propensity score matching, which showed that patients' age, DEX infusion, comorbidity of diabetes, and preoperative serum phosphorus were independent factors for SHPT recurrence, and that patients who received DEX had an estimated 3.80-fold decrease in the risk of SHPT recurrence (OR, 0.263; 95\% $\mathrm{Cl}, 0.081$ to $0.854 ; P=0.026)$. 
Table 2

Association between dexmedetomidine and postoperative recurrence in patients with tPTX-AT surgery after multivariable logistic analyses with propensity score matching.

\begin{tabular}{|c|c|c|c|c|c|c|c|}
\hline \multirow[t]{2}{*}{ Parameters } & & \multicolumn{3}{|c|}{ Univariate analysis } & \multicolumn{3}{|c|}{ Multivariable analysis } \\
\hline & & OR & $95 \% \mathrm{Cl}$ & $\begin{array}{l}\mathrm{P}- \\
\text { value }\end{array}$ & OR & $95 \% \mathrm{Cl}$ & $\begin{array}{l}\mathrm{P} \text { - } \\
\text { value }\end{array}$ \\
\hline \multirow[t]{5}{*}{ Age (years) } & $<40$ & 1.000 & ref & & & & \\
\hline & 40 to 50 & 0.606 & $\begin{array}{l}0.156 \text { to } \\
2.359\end{array}$ & 0.470 & 0.456 & $\begin{array}{l}0.094 \text { to } \\
2.211\end{array}$ & 0.330 \\
\hline & 50 to 60 & 1.050 & $\begin{array}{l}0.292 \text { to } \\
3.775\end{array}$ & 0.940 & 0.998 & $\begin{array}{l}0.219 \text { to } \\
4.555\end{array}$ & 0.998 \\
\hline & 60 to 70 & 4.500 & $\begin{array}{l}1.125 \text { to } \\
17.99\end{array}$ & 0.033 & 10.291 & $\begin{array}{l}1.778 \text { to } \\
59.546\end{array}$ & 0.009 \\
\hline & $\geq 70$ & 24.000 & $\begin{array}{l}1.768 \text { to } \\
325.783\end{array}$ & 0.017 & 161.745 & $\begin{array}{l}3.123 \text { to } \\
8376.977\end{array}$ & 0.012 \\
\hline Female & & 0.850 & $\begin{array}{l}0.358 \text { to } \\
2.017\end{array}$ & 0.712 & 0.560 & $\begin{array}{l}0.187 \text { to } \\
1.672\end{array}$ & 0.298 \\
\hline \multirow[t]{4}{*}{$\mathrm{BMI}\left(\mathrm{kg} \mathrm{m}^{-2}\right)$} & $<18.5$ & 1.000 & ref & & 1.000 & ref & \\
\hline & 18.5 to 25 & 0.618 & $\begin{array}{l}0.190 \text { to } \\
2.004\end{array}$ & 0.422 & 0.374 & $\begin{array}{l}0.089 \text { to } \\
1.569\end{array}$ & 0.179 \\
\hline & 25 to 30 & 1.120 & $\begin{array}{l}0.291 \text { to } \\
4.343\end{array}$ & 0.864 & 0.730 & $\begin{array}{l}0.136 \text { to } \\
3.904\end{array}$ & 0.713 \\
\hline & $\geq 30$ & N/A & & 0.999 & & & \\
\hline \multirow[t]{3}{*}{ Comorbidities } & Hypertension & 0.615 & $\begin{array}{l}0.215 \text { to } \\
1.758\end{array}$ & 0.364 & 0.556 & $\begin{array}{l}0.163 \text { to } \\
1.892\end{array}$ & 0.347 \\
\hline & Diabetes & 3.606 & $\begin{array}{l}0.686 \text { to } \\
18.943\end{array}$ & 0.130 & 9.282 & $\begin{array}{l}1.006 \text { to } \\
85.642\end{array}$ & 0.049 \\
\hline & $\begin{array}{l}\text { Cardiopulmonary } \\
\text { disease }\end{array}$ & 2.374 & $\begin{array}{l}0.482 \text { to } \\
11.678\end{array}$ & 0.288 & 0.309 & $\begin{array}{l}0.017 \text { to } \\
5.698\end{array}$ & 0.430 \\
\hline \multirow[t]{2}{*}{ ASA physical status } & ? & 1.000 & ref & & & & \\
\hline & $\square$ & $\mathrm{N} / \mathrm{A}$ & & 0.999 & & & \\
\hline \multirow[t]{2}{*}{ Type of dialysis } & Hemodialysis & 1.000 & ref & & & & \\
\hline & Peritoneal dialysis & $\mathrm{N} / \mathrm{A}$ & & 1.000 & & & \\
\hline $\begin{array}{l}\text { Dialysis duration } \\
\text { (years) }\end{array}$ & & 1.063 & $\begin{array}{l}0.923 \text { to } \\
1.224\end{array}$ & 0.398 & 1.175 & $\begin{array}{l}0.975 \text { to } \\
1.417\end{array}$ & 0.090 \\
\hline $\begin{array}{l}\text { Duration of } \\
\text { anesthesia (min) }\end{array}$ & & 0.991 & $\begin{array}{l}0.980 \text { to } \\
1.002\end{array}$ & 0.103 & 0.988 & $\begin{array}{l}0.975 \text { to } \\
1.002\end{array}$ & 0.089 \\
\hline
\end{tabular}

Propensity score matching analyses were adjusted by factors in Table 1. Parameters of gender, BMI, and values with $P$-values $<0.5$ in univariate analysis were subjected to multivariable analyses.

Abbreviations: tPTX-AT, parathyroidectomy with auto-transplantation; BMI, body mass index; ASA, American Society of Anesthesiologist; Ca, calcium; P, phosphorus; iPTH, intact parathyroid hormone; ALP, alkaline phosphatase; OR, odds ratio; $\mathrm{Cl}$, confidence interval; ref, reference. 


\begin{tabular}{|c|c|c|c|c|c|c|c|}
\hline \multirow[t]{2}{*}{ Parameters } & & \multicolumn{3}{|c|}{ Univariate analysis } & \multicolumn{3}{|c|}{ Multivariable analysis } \\
\hline & & OR & $95 \% \mathrm{Cl}$ & $\begin{array}{l}\text { P- } \\
\text { value }\end{array}$ & OR & $95 \% \mathrm{Cl}$ & $\begin{array}{l}\mathrm{P} \text { - } \\
\text { value }\end{array}$ \\
\hline \multirow[t]{2}{*}{ Year at surgery } & 2017 & 1.000 & ref & & 1.000 & ref & \\
\hline & 2018 & 0.630 & $\begin{array}{l}0.266 \text { to } \\
1.495\end{array}$ & 0.295 & 0.812 & $\begin{array}{l}0.266 \text { to } \\
2.478\end{array}$ & 0.714 \\
\hline \multirow[t]{4}{*}{$\begin{array}{l}\text { Preoperative } \\
\text { measures }\end{array}$} & Serum Ca $\left(\mathrm{mg} \mathrm{dl}^{-1}\right)$ & 2.060 & $\begin{array}{l}0.429 \text { to } \\
9.889\end{array}$ & 0.367 & 1.194 & $\begin{array}{l}0.196 \text { to } \\
7.283\end{array}$ & 0.847 \\
\hline & Serum $\mathrm{P}\left(\mathrm{mg} \mathrm{dl}^{-1}\right)$ & 1.340 & $\begin{array}{l}0.625 \text { to } \\
2.875\end{array}$ & 0.452 & 3.167 & $\begin{array}{l}1.191 \text { to } \\
8.421\end{array}$ & 0.021 \\
\hline & $\begin{array}{l}\text { Serum iPTH (pg } \\
\left.\mathrm{ml}^{-1}\right)\end{array}$ & 1.000 & $\begin{array}{l}1.000 \text { to } \\
1.001\end{array}$ & 0.418 & 1.000 & $\begin{array}{l}0.999 \text { to } \\
1.001\end{array}$ & 0.930 \\
\hline & Serum ALP $\left(U L^{-1}\right)$ & 1.000 & $\begin{array}{l}0.999 \text { to } \\
1.001\end{array}$ & 0.984 & & & \\
\hline $\begin{array}{l}\text { Intraoperative } \\
\text { measures }\end{array}$ & $\begin{array}{l}\text { Dexmedetomidine } \\
\text { infusion }\end{array}$ & 0.343 & $\begin{array}{l}0.124 \text { to } \\
0.947\end{array}$ & 0.039 & 0.263 & $\begin{array}{l}0.081 \text { to } \\
0.854\end{array}$ & 0.026 \\
\hline \multicolumn{8}{|c|}{$\begin{array}{l}\text { Propensity score matching analyses were adjusted by factors in Table } 1 \text {. Parameters of gender, BMI, and values } \\
\text { with } P \text {-values }<0.5 \text { in univariate analysis were subjected to multivariable analyses. }\end{array}$} \\
\hline
\end{tabular}

\section{Comparison of outcomes between patients did and did not receive DEX}

Patients who received DEX consumed less analgesics during the surgery. Specifically, sufentanil consumption in the DEX group was significantly decreased compared with that in the CON group $(25.6 \pm 7.1 \mu \mathrm{g} v s 28.1 \pm 4.9 \mu \mathrm{g}, P=0.001)$. Meanwhile, DEX provided better pain control postoperatively. The number of patients with a pain numerical rating scale (0 to 10) $\geq 4$ in the PACU in the DEX group was significantly lower than that in the CON group. However, DEX resulted in a lower heart rate postoperatively, which was treated with atropine accordingly, and no severe hemodynamic instability occurred. There were no significant differences between the two groups in terms of the risk of postoperative nausea and vomiting, hypertension, and hypotension after the surgery, nor in the length of hospital stay after surgery and hospitalisation costs (Table 3). One year later, patients who received DEX exhibited a better QOL in terms of physical functioning, general health perception, and less emotional role limitation. However, there was no significant difference in the other items of the SF-36 assessment (Fig. 2). 
Table 3

Postoperative parameters of patients who received dexmedetomidine (DEX group) and those who did not receive dexmedetomidine (CON group).

\begin{tabular}{|c|c|c|c|c|c|c|}
\hline \multirow[t]{3}{*}{ Parameters } & \multicolumn{3}{|c|}{ Before propensity score matching $(n=354)$} & \multicolumn{3}{|c|}{$\begin{array}{l}\text { After } 1: 2 \text { propensity score matchining } \\
(n=268)\end{array}$} \\
\hline & CON group & DEX group & $P$-value & CON group & DEX group & $P$-value \\
\hline & $n=221$ & $n=133$ & & $n=157$ & $n=111$ & \\
\hline $\begin{array}{l}\text { Sufentanil } \\
\text { consumption } \\
(\mu \mathrm{g})\end{array}$ & $27.9 \pm 5.3$ & $25.6 \pm 7.1$ & $0.001^{\mathrm{ad}}$ & $28.1 \pm 4.9$ & $25.6 \pm 7.1$ & $0.001^{a c}$ \\
\hline $\begin{array}{l}\text { Extubation } \\
\text { time (min) }\end{array}$ & $5.0[5.0$ to 7.0$]$ & $5.0[4.0$ to 7.5$]$ & $0.337^{b}$ & $5.0[4.5$ to 7.0$]$ & $5.0[4.0$ to 8.0$]$ & $0.741^{b}$ \\
\hline $\begin{array}{l}\text { PACU time } \\
(\min )\end{array}$ & $\begin{array}{l}50.0[40.0 \text { to } \\
64.5]\end{array}$ & $\begin{array}{l}48.0[36.0 \text { to } \\
63.0]\end{array}$ & $0.216^{b}$ & $\begin{array}{l}53.0[41.0 \text { to } \\
65.0]\end{array}$ & $\begin{array}{l}48.0[38.0 \text { to } \\
63.0]\end{array}$ & $0.262^{b}$ \\
\hline
\end{tabular}

Adverse Events

during PACU

\begin{tabular}{|c|c|c|c|c|c|c|}
\hline$V A S \geq 4$ & $35(15.8)$ & $11(8.3)$ & $0.067^{c}$ & $32(20.4)$ & $11(9.9)$ & $0.021^{\mathrm{cd}}$ \\
\hline Hypertension & $40(18.1)$ & $15(11.3)$ & $0.086^{c}$ & $31(19.7)$ & 15(13.5) & $0.183^{c}$ \\
\hline Hypotension & $0(0)$ & $2(1.5)$ & $0.140^{c}$ & $0(0)$ & $2(1.8)$ & $0.171^{c}$ \\
\hline Bradycardia & $14(6.3)$ & 19(14.3) & $0.013^{\mathrm{cd}}$ & $10(6.4)$ & 17(15.3) & $0.017^{\mathrm{cd}}$ \\
\hline Tachycardia & $26(11.8)$ & $8(6.0)$ & $0.075^{c}$ & 21(13.4) & $6(5.4)$ & $0.033^{\mathrm{cd}}$ \\
\hline PONV & $4(1.8)$ & $5(3.8)$ & $0.305^{c}$ & $3(1.9)$ & $5(4.5)$ & $0.282^{c}$ \\
\hline \multicolumn{7}{|l|}{$\begin{array}{l}\text { Postoperative } \\
\text { measures at } \\
\text { discharge }\end{array}$} \\
\hline $\begin{array}{l}\text { Serum Ca (mg } \\
\mathrm{dl}^{-1} \text { ) }\end{array}$ & $2.07 \pm 0.3$ & $2.08 \pm 0.33$ & $0.913^{a}$ & $2.08 \pm 0.33$ & $2.07 \pm 0.32$ & $0.751^{a}$ \\
\hline $\begin{array}{l}\text { Serum P (mg } \\
\left.\mathrm{dl}^{-1}\right)\end{array}$ & $1.98 \pm 0.58$ & $1.92 \pm 0.50$ & $0.350^{a}$ & $1.97 \pm 0.56$ & $1.91 \pm 0.47$ & $0.353^{a}$ \\
\hline 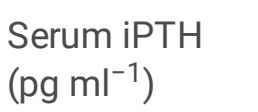 & 3.0 [3.0 to 8.2] & 3.0 [3.0 to 9.6$]$ & $0.930^{\mathrm{b}}$ & $\begin{array}{l}3.0[3.0 \text { to } \\
10.0]\end{array}$ & $3.0[3.0$ to 6.0$]$ & $0.658^{b}$ \\
\hline
\end{tabular}

Data are presented as the mean \pm SD or median [IQR] for continuous variables, and number (\%) for categorical variables.

Abbreviations: PACU, post-anesthesia care unit; VAS, visual analog score; PONV, post-operative nausea and vomiting; Ca, calcium; P, phosphorus; iPTH, intact parathyroid hormone.

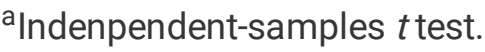

${ }^{\mathrm{b}}$ Rank sum test.

'Pearson's $\chi^{2}$ test.

${ }^{\mathrm{d}} P<0.05$ versus control. 


\begin{tabular}{|c|c|c|c|c|c|c|}
\hline \multirow{2}{*}{$\begin{array}{l}\text { Parameters } \\
\text { Length of } \\
\text { hospital stay } \\
\text { after surgery } \\
\text { (days) }\end{array}$} & \multicolumn{3}{|c|}{ Before propensity score matching(n=354) } & \multicolumn{3}{|c|}{$\begin{array}{l}\text { After } 1: 2 \text { propensity score matchining } \\
(n=268)\end{array}$} \\
\hline & $5.7 \pm 3.8$ & $5.2 \pm 2.6$ & $0.170^{\mathrm{a}}$ & $5.7 \pm 4.0$ & $5.3 \pm 2.7$ & $0.336^{a}$ \\
\hline $\begin{array}{l}\text { Hospitalization } \\
\text { cost (US } \\
\text { dollars) }\end{array}$ & $4038.6 \pm 1213.4$ & $4065.1 \pm 1163.0$ & $0.840^{\mathrm{a}}$ & $4098.0 \pm 1217.5$ & $4067.9 \pm 1163.2$ & $0.839^{a}$ \\
\hline \multicolumn{7}{|c|}{$\begin{array}{l}\text { Data are presented as the mean } \pm \text { SD or median [IQR] for continuous variables, and number }(\%) \text { for categorical } \\
\text { variables. }\end{array}$} \\
\hline \multicolumn{7}{|c|}{$\begin{array}{l}\text { Abbreviations: PACU, post-anesthesia care unit; VAS, visual analog score; PONV, post-operative nausea and } \\
\text { vomiting; Ca, calcium; P, phosphorus; iPTH, intact parathyroid hormone. }\end{array}$} \\
\hline \multicolumn{7}{|c|}{${ }^{\mathrm{a}}$ Indenpendent-samples $t$ test. } \\
\hline \multicolumn{7}{|l|}{ brank sum test. } \\
\hline \multicolumn{7}{|c|}{ 'Pearson's $\chi^{2}$ test. } \\
\hline
\end{tabular}

Part of the CPR concentration of patients were missing during the follow-up. The median [IQR] of CRP concentration were 80.4 [55.9 to 104.1] mg L $\mathrm{m}^{-1}$ in DEX group ( $\mathrm{n}=130$, with 3 missing), and 89.5 [61.3 to 119.8] $\mathrm{mg} \mathrm{L}^{-1}$ in CON group ( $n=65$, with 156 missing). Patients in the DEX group exhibited lower CRP concentration compared with those in CON group one year after surgery.

\section{Discussion}

In this analysis of consecutive patients with end-stage renal disease (ESRD) undergoing tPTX-AT at our institution, we found that intraoperative DEX use was associated with a lower risk of SHPT recurrence. Meanwhile, patients who received DEX exhibited a higher health-related QOL one year after the surgery.

Parathyroidectomy with Auto-Transplantation involves the removal of all four parathyroid glands and the implantation of a section of one of the glands into a muscle, which leaves enough residual parathyroid tissue to support mineral homoeostasis. It has been reported that tPTX-AT has a high success rate and a slightly lower risk of hyperparathyroidism recurrence $[13,14]$. During the data collection, we chose the patients who were operated by the same surgical team to exclude the influence of operative procedures on the results. The iPTH concentrations tested at discharge were all decreased to the normal level, which indicated a successful operation. However, because the pathogenic factors may persist and the residual parathyroid tissue may still show increased proliferation of parathyroid cells, SHPT recurrence remains a significant problem in the treatment of nephrogenic hyperparathyroidism.

Increased inflammation has been proposed to play an important role in the pathogenesis of multiple diseases contributing to a low QOL in dialysis patients [15]. Compared with uremic patients without SHPT, patients with SHPT may have a higher prevalence of serum inflammatory cytokines, such as CRP, which is an acute phase protein, and is most often used as an inflammatory biomarker in nephrology [16]. Additionally, surgical trauma induces a variety of stress responses and further aggravate perioperative inflammation [8]. Therefore, controlling the inflammatory response perioperatively could help inhibit hyperfunction of parathyroid cells. Clinical studies have shown that DEX can attenuate perioperative stress and inflammation induced by surgical trauma and exhibit multifaceted protective effects when 
administered as an adjuvant [17]. It was reported that patients receiving DEX perioperatively had a significant decrease in CRP concentration 24 to $48 \mathrm{~h}$ after surgery compared with those who did not $[18,19]$. In the current study, CRP concentrations were also collected from some patients, although some patients had missing data. Patients in the DEX group exhibited lower CRP concentrations compared with the CON group postoperatively. This partly indicated an association of DEX with reduced inflammation, which may have resulted in a lower risk of SHPT recurrence. However, this anti-inflammatory mechanism should be explored further with a larger and integral sample size or prospective studies in the future.

We also found that infusion of DEX decreased opioid consumption intraoperatively and enhanced postoperative pain control for patients in the PACU. Previous studies have demonstrated that the opioid receptor antagonist, naloxone, had a suppressive effect on iPTH in patients with renal failure [20]. Therefore, we speculated that reduced opioid consumption may similarly lead to the inhibition of hyperfunction in parathyroid cells and decrease the risk of SHPT recurrence. Meanwhile, with the development of opioid-sparing anesthesia, patients exhibit a better prognosis for multiple surgeries [21, 22]. As we know, patients with ESRD usually experience substantial physical, emotional, mental, and psychological impairments that are reflected in the decreased QOL $[13,23]$. In the current study, DEX demonstrated a beneficial effect on the QOL in dialysis patients after TPTX-AT, and significant improvements were observed in physical functioning, general health, and role limitations due to emotional problems, which may be an indirect result of the opioid-sparing effects of DEX.

In addition to DEX infusion, we found three independent risk factors associated with SHPT recurrence: higher age, comorbidity of diabetes mellitus, and high preoperative phosphorus concentrations. Elderly patients are more likely to represent an extreme model for arteriosclerosis, vascular calcification, and bone disorders with the progression of ESRD. Additionally, these pathological features are also relevant in other common chronic health abnormalities, such as diabetes mellitus and chronic inflammatory and electrolyte disturbances [24]. Thus, patients with higher age, comorbidity of diabetes mellitus, and high preoperative phosphorus concentrations may be vulnerable to SHPT recurrence.

There were some important limitations to this study. First, our study was limited by its relatively small sample size. Nevertheless, this is the largest study to assess the effect of DEX on SHPT recurrence after tPTX-AT. Second, as for any retrospective study, unknown confounding factors were a major limitation. Although our ASD analysis was based on preoperative baseline data, some other variables might have still differed between the patients who did and did not receive DEX. For instance, CRP data was incomplete, and we did not consider the consumption of anesthetics, hemodynamics, and the depth of anesthesia intraoperatively. However, we adjusted for the anesthetic time in the multivariable analysis of unmatched and matched patients. Third, this was a single-centre study, which may have compromised the generalisability of the findings, and the retrospective observational study design may have resulted in selection bias. Nevertheless, a large number of patients with SHPT visit our hospital; therefore, collecting data on the current cases of tPTX-AT for SHPT in a prospective registry would aid in future outcome analyses and high-quality research.

\section{Conclusion}

In conclusion, our clinical data suggest that intraoperative use of DEX is associated with a lower risk of SHPT recurrence in uremic patients undergoing IPTX-AT, and that DEX use can improve the QOL in this population. We believe that the use of DEX as an adjuvant in general anesthesia contributes positively to the prognosis of uremic patients.

Prospective randomized controlled trials are needed to accurately assess the effects of DEX on the prognosis of patients with ESRD undergoing TPTX-AT, and basic research should further elucidate the potential mediating mechanisms in this population.

Page $11 / 15$ 


\section{Declarations}

\section{Acknowledgements}

This work was supported by the National Natural Science Foundation of China (grant number: 81900316 to YW)

\section{Authors contributions}

Y.W., W.H. and Y.Z. designed the study; Y.W., W.H. and P.L. collected and interpreted the data; W.H. and X.H analyzed the data, and prepared accompanying figures and material; Y.W. drafted the manuscript; Y.Z. revised and finalized the manuscript. All authors reviewed and approved the submitted version.

\section{Competing interests}

The authors declare no competing interest.

\section{References}

1. Slatopolsky, E., Brown, A. \& Dusso, A. Pathogenesis of secondary hyperparathyroidism. Kidney Int Suppl, 73, S1419 (1999).

2. Rodriguez, M. \& Lorenzo, V. Parathyroid hormone, a uremic toxin. Semin Dial, 22, 363-368 (2009).

3. Steffen, L., Moffa, G., Müller, P. C. \& Oertli, D. Secondary hyperparathyroidism: recurrence after total parathyroidectomy with autotransplantation. Swiss Med Wkly, 149, w20160 (2019).

4. Hou, J. et al. Network meta-analysis of surgical treatment for secondary hyperparathyroidism. Am J Otolaryngol, 41, 102370 (2020).

5. Wall, T., Sherwin, A., Ma, D. \& Buggy, D. J. Influence of perioperative anaesthetic and analgesic interventions on oncological outcomes: a narrative review. Br J Anaesth, 123, 135-150 (2019).

6. Kim, M. H. et al. Tramadol use is associated with enhanced postoperative outcomes in breast cancer patients: a retrospective clinical study with in vitro confirmation. Br J Anaesth, 123, 865-876 (2019).

7. Xia, M. et al. Dexmedetomidine regulate the malignancy of breast cancer cells by activating a2-adrenoceptor/ERK signaling pathway. Eur Rev Med Pharmacol Sci, 20, 3500-3506 (2016).

8. Li, Y. et al. Dexmedetomidine Combined with General Anesthesia Provides Similar Intraoperative Stress Response Reduction When Compared with a Combined General and Epidural Anesthetic Technique. Anesth Analg, 122, 1202-1210 (2016).

9. Ferreira, J. A. \& Bissell, B. D. Misdirected Sympathy: The Role of Sympatholysis in Sepsis and Septic Shock. J Intensive Care Med, 33, 74-86 (2018).

10. Li, Q., Chen, C., Chen, X., Han, M. \& Li, J. Dexmedetomidine attenuates renal fibrosis via a2-adrenergic receptordependent inhibition of cellular senescence after renal ischemia/reperfusion. Life Sci, 207, 1-8 (2018).

11. Yu, J., Coons, S. J., Draugalis, J. R., Ren, X. S. \& Hays, R. D. Equivalence of Chinese and US-English versions of the SF-36 health survey. Qual Life Res, 12, 449-457 (2003).

12. Ware, J. E. J. \& Sherbourne, C. D. The MOS 36-item short-form health survey (SF-36). I. Conceptual framework and item selection. Med Care, 30, 473-483 (1992).

13. Sun, Y., Cai, H., Bai, J., Zhao, H. \& Miao, Y. Endoscopic total parathyroidectomy and partial parathyroid tissue autotransplantation for patients with secondary hyperparathyroidism: a new surgical approach. World J Surg, 33, 1674-1679 (2009). 
14. Albuquerque, R. C. et al. Parathyroidectomy in patients with chronic kidney disease: Impacts of different techniques on the biochemical and clinical evolution of secondary hyperparathyroidism. Surgery, 163, 381-387 (2018).

15. Patel, T. V. \& Singh, A. K. Kidney disease outcomes quality initiative guidelines for bone and mineral metabolism: emerging questions. Semin Nephrol, 29, 105-112 (2009).

16. Tentori, F. et al. Mortality risk for dialysis patients with different levels of serum calcium, phosphorus, and PTH: the Dialysis Outcomes and Practice Patterns Study (DOPPS). Am J Kidney Dis, 52, 519-530 (2008).

17. Wang, K. et al. Effects of dexmedetomidine on perioperative stress, inflammation, and immune function: systematic review and meta-analysis. Br J Anaesth, 123, 777-794 (2019).

18. Dong, W. et al. The effect of dexmedetomidine on expressions of inflammatory factors in patients with radical resection of gastric cancer. Eur Rev Med Pharmacol Sci, 21, 3510-3515 (2017).

19. Wang, K. \& Li, C. Effects of dexmedetomidine on inflammatory factors, T lymphocyte subsets and expression of NF-KB in peripheral blood mononuclear cells in patients receiving radical surgery of colon carcinoma. Oncol Lett, 15, 7153-7157 (2018).

20. Grzeszczak, W., Kokot, F. \& Duława, J. Effects of naloxone administration on endocrine abnormalities in chronic renal failure. Am J Nephrol, 7, 93-100 (1987).

21. Shim, H. \& Gan, T. J. Side effect profiles of different opioids in the perioperative setting: are they different and can we reduce them. Br J Anaesth, 123, 266-268 (2019).

22. Awada, H. N. et al. Postoperative cognitive dysfunction is rare after fast-track hip- and knee arthroplasty - But potentially related to opioid use. J Clin Anesth, 57, 80-86 (2019).

23. Cheng, S. P. et al. Parathyroidectomy improves symptomatology and quality of life in patients with secondary hyperparathyroidism. Surgery, 155, 320-328 (2014).

24. Covic, A. et al. Bone and mineral disorders in chronic kidney disease: implications for cardiovascular health and ageing in the general population. Lancet Diabetes Endocrinol, 6, 319-331 (2018).

\section{Figures}




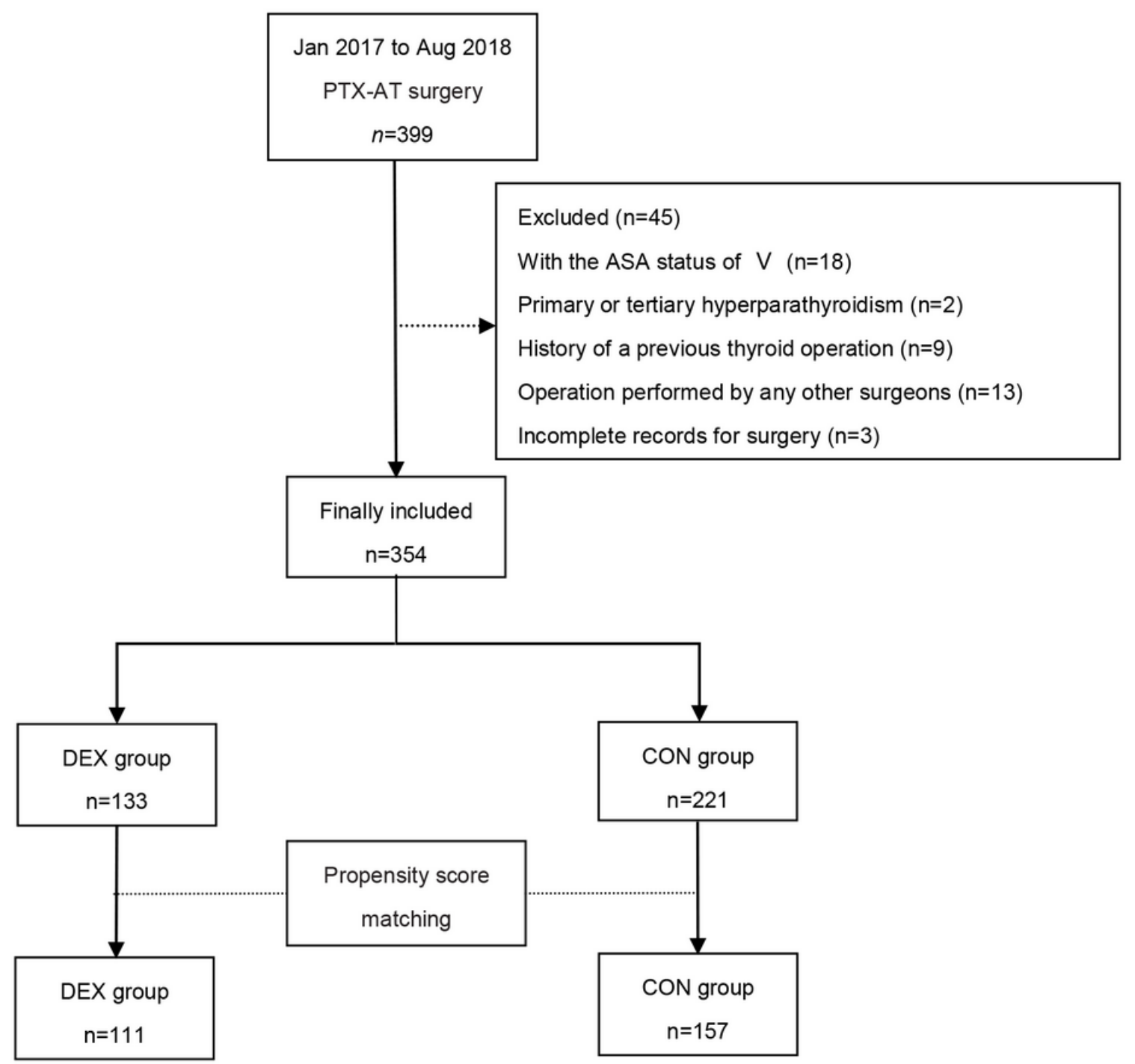

Figure 1

Flow chart of patient selection. tPTX-AT, parathyroidectomy with auto-transplantation; ASA, American Society of Anesthesioligists; DEX, dexmedetomidine group; CON, control group

a.

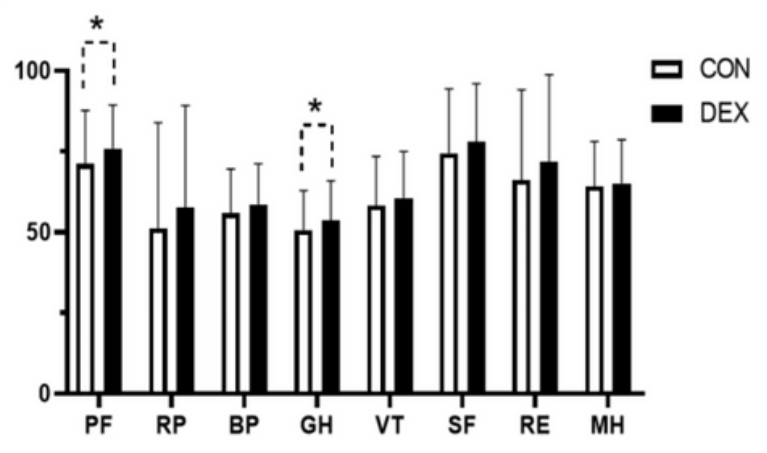

b.

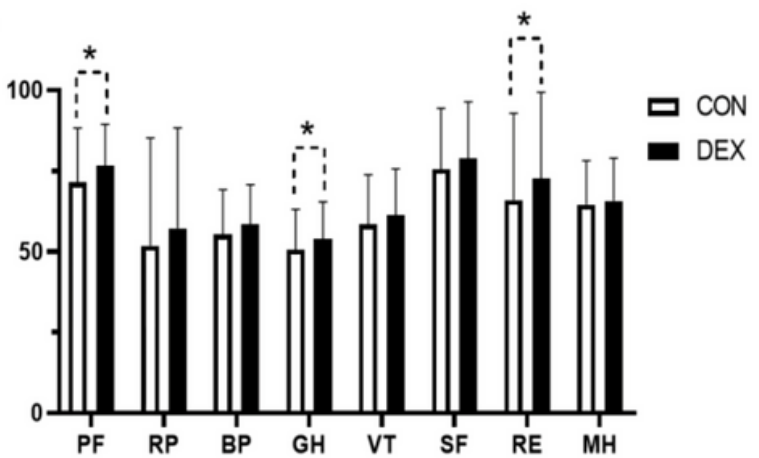

Figure 2 
Scores of Short Form (SF)-36 items of patients one year after surgery. a. Patients received dexmedetomidine exhibited a significantly higher score in terms of physical functioning (PF) and general health (GH) before propensity score matching (PF: $75.86 \pm 13.52$ vs $71.11 \pm 16.56$; $\mathrm{GH}: 53.74 \pm 12.19$ vs $50.71 \pm 12.17$ ); b. In addition to a significantly higher score in the PF and GH (PF:76.58 \pm 12.90 vs $71.50 \pm 16.79 ; \mathrm{GH}: 53.91 \pm 11.48$ vs $50.71 \pm 12.17)$, patients who received dexmedetomidine also exhibited less emotional role limitation ( $R E$ ) after propensity score matching (72.67 \pm 26.67 vs $50.71 \pm 12.17)$. Data were compared by independent-samples $t$ test. ${ }^{*} P<0.05$ compared between the two groups. CON, control group; DEX, dexmedetomidine group; PF, physical functioning; RP, physical role limitation, BP, bodily pain; $\mathrm{GH}$, general health perception; VT, vitality; SF, social functioning; RE, emotional role limitation; $\mathrm{MH}$, mental health. 